\title{
Interface instability in the bulk processing of 2223 BSCCO powders
}

\author{
By Ming Dao†‡, Robert J. Asaro †† and Robert J. Sebring§ \\ †Trans-Science Corporation, 7777 Fay Avenue, Suite 112, La Jolla, \\ California 92037, USA \\ $\$$ Division of Structural Engineering, 0085, University of California, San Diego, \\ La Jolla, California 92093, USA \\ $\S$ Materials Science and Technology Division Los Alamos National Labortory, \\ MST-5, MS G730, Los Alamos, New Mexico 87545, USA
}

[Received 25 September 1997 and accepted in revised form 28 December 1997]

\begin{abstract}
Interface instability (or 'sausaging') has been a major problem in the oxide powder-in-tube tape-rolling process. The fast Fourier transform of core instabilities are first performed to study the rolled tapes and to obtain quantitative information on the wavelengths and magnitudes of the nonuniform profiles. The existing experimental observations are also re-examined and summarized. Treating this problem as the bimaterial interface instability, both bifurcation analysis and finite-element modelling are applied to study the influences of roll-gap geometry, packing fill factor and clad material properties on the instability wavelength and magnitude. Good correlations between theoretical results and experimental observations are found. The critical wavelength/current core thickness ratio $\lambda^{*} / d_{\mathrm{c}}$ is found to be sensitive to the fill factor only, and insensitive to the reduction/pass ratio, and the core and clad material properties; that is, the relative tape geometry is the single dominating factor that affect the normalized critical wavenumber $\lambda^{*} / d_{\mathrm{c}}$. Consistent with experimental observations, a smaller reduction/pass ratio, a higher initial core porosity, a higher hardening clad material and a larger core fill factor can reduce the normalized instability magnitude $\Delta v / d_{c}$ (i.e. the instability magnitude/current core thickness ratio) at the same tape reduction strain level. The results suggest that the reason for the much smaller interface variation magnitude with a small reduction/pass ratio (i.e. 5\% per pass) compared with a large reduction/pass ratio (i.e. $25 \%$ per pass) is not because the small reduction/ pass ratio can eliminate or delay the interface instability initiation; it is, however, most probably caused by the random disruption of the interface by the many rolling steps with critical wavelengths very close to each other between adjacent rolling steps.
\end{abstract}

\section{$\S 1$. Introduction}

Developing long-length high- $J_{\mathrm{c}}$ superconducting tapes has been a major worldwide effort in recent years because of their potential applications in power transmission lines, motors and other devices. Applications of high-temperature superconducting oxides, in either bulk or thin-film form, require synthesis and processing that lead to chemically, structurally and electronically optimal microstructures. The attainment of such optimal microstructures hinges upon achieving, at the very least, firstly a high degree of densification, secondly sharp crystallographic textures characterized by a high degree of alignment of superconducting crystal planes lying parallel to the conducting direction and thirdly a minimal density of cracks whose 
faces 'block' the flow of current. The need for strong microstructural control is due to the inherently anisotropic structure, and the resulting properties of these oxides which, for example, tend to superconduct preferentially in the c plane. This class of oxides tends to be brittle and thus difficult to process by conventional means. Some earlier research has been concerned with developing a basic understanding of their mechanical behaviour, using the knowledge obtained to build a quantitative theory of deformation and failure of these materials, and implementing these design synthesis and processing methods to predict the microstructures that result from them (Asaro et al. 1992, Ahzi et al. 1993, Schoenfeld et al. 1996a). Optimal microstructures have been described by Asaro et al. (1992) and Ahzi et al. (1993) using polycrystal plasticity averaging schemes. Numerical simulations were performed to calculate inelastic deformation due to crystallographic slip on the limited number of slip systems available within the single crystals of $\mathrm{Bi}_{2} \mathrm{Sr}_{2} \mathrm{Ca}_{2} \mathrm{Cu}_{3} \mathrm{O}_{x}(\mathrm{BSCCO} 2223)$ superconductors and to sliding following atomic debonding on the $\mathbf{a}$ and $\mathbf{b}$ planes of the orthorhombic unit cell of BSCCO 2223 crystals. Deformation textures, as well as stress-strain behaviour for a broad range of low-symmetry crystal aggregates have been modelled using this particular constitutive theory (Parks and Ahzi 1990, Lee et al. 1993, Schoenfeld et al. 1995).

As previously noted, the mechanical processing of such oxides begins by densification from a powder. The powder is hand packed into a silver tube. The tube is then drawn and subsequently rolled to achieve both high densification and a high degree of crystallographic texture. Figure 1 shows a schematic drawing of the taperolling process. Problems that arise during such processing include, but are not limited to firstly poor densification, secondly the coalescence of microcracks and residual stress states leading to macroscopic cracking and thirdly instabilities in the oxide core during rolling which to lead to a 'sausaging' of the core (for example Parrell et al. (1993)). The influence of the deformation parameters on the density of BSCCO filaments as well as on the creation of sausaging and cracks was described in Han et al. (1997), Husek et al. (1995), Korzekwa et al. (1994), Fischer et al. (1995),

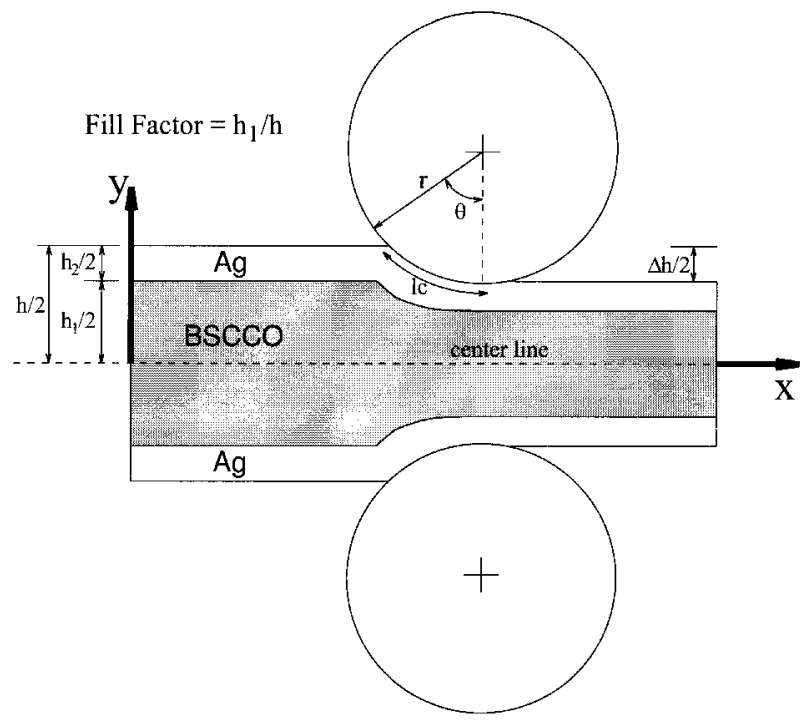

Figure 1. Schematic drawing of the tape-rolling process. 
and Karuna et al. (1995). The influence of silver alloy sheaths, which are stronger than the pure silver ones, on the deformation homogeneity was studied experimentally in Tanaka et al. (1995), and Ahn et al. (1994). Schoenfeld et al. (1996a) modelled the densification problem coupled with crystallographic texturing, and Schoenfeld et al. (1996b) studied the $\mathrm{Bi}-\mathrm{Sr}-\mathrm{Ca}-\mathrm{Cu}-\mathrm{O}$ (BSCCO) powder tape-rolling process.

The previous studies have made major advances in predicting deformation textures in such materials, as well as in understanding the controlling and optimizing processing parameters for this oxide powder-in-tube (OPIT) tape rolling process. In this, geometrical non-uniformities have been linked to lower current densities (Parrell et al. 1993). However, much work needs to be done to understand the core instabilities (or 'sausaging' effects) during the tape rolling.

The highest degree of powder densification and textural alignment depend on pushing the limits of powder compaction during the final cold working of the twophase composite. Further, efficient use of the oxide is also made by rolling the thinnest tapes possible, that is, the thinner the tape that can be rolled, the longer it becomes, thus increasing the lengths of tapes that are possible. Probing the limits to which rolling can be achieved will help to maximize these parameters. The limiting thickness of such tapes seems to be controlled to a high degree by instabilities in the oxide core. Such instabilities can be either 'sausaging' modes (see figure 2 later) or by shear localization leading to fracture (see figure 6 later).

To study this problem, we first apply numerical techniques to analyse quantitatively the frequencies and magnitudes of the sausaging effects. Next, analytic methods using bifurcation analyses (for example Steif (1987)) are applied to study the instability wavelength and compressive strains of such interface instability modes, where the problem is treated as plane-strain compression of a two-phase composite. Finite-element analysis is then performed on the OPIT rolling process to analyse the wavelength and magnitude of the possible instabilities. Finally, the bifurcation results and finite-element computations are compared with the experimental results.

\section{§2. Experimental perspectives and fast Fourier transform}

FREQUENCY ANALYSES

Rolling experiments have been conducted specifically to investigate the effects of process parameters on core uniformity within the composite workpiece. Since the goal of this particular work is to investigate such uniformity, no attempt was made to measure the electronic properties of the resulting tape, only to correlate process parameters with mechanical properties and the overall tape quality within the context of rolling a highly dense uniform core. Subsequent fast Fourier transformations were performed to document and categorize the frequencies and magnitudes of the uniformity of the cores.

Tubes of either 0.9999 fine silver or 1100 (commercial purity) aluminium were packed with $\mathrm{BSCCO} 2223$ precursor powder consisting of $\mathrm{Bi}_{2} \mathrm{Sr}_{2} \mathrm{CaCu}_{2} \mathrm{O}_{x}, \mathrm{Ca}_{2} \mathrm{CuO}_{3}$ and $\mathrm{CuO}$ of stoichiometric quantities. The mean particle size was $4.7 \mu \mathrm{m}$ with a distribution of $\pm 1.2 \mu \mathrm{m}$

Silver-sheathed BSCCO filled tapes at $60 \%$ fill factors with two different rolling reduction schedules were studied in detail. The first was a $25 \%$ or large reduction/ pass ratio and the second a $5 \%$ or small reduction/pass ratio. Eight samples were taken from each rolling experiment. One sample for fast Fourier transform (FFT) analysis was taken after each large reduction pass. For the small-reduction series, a tape sample for analysis was taken when the tape thickness, as measured with a 
caliper, was close to the tape thickness of the large-reduction samples. Thus there were eight small-reduction samples that corresponded in total tape thickness to each one of the eight large-reduction samples, but there were several reductions between each small reduction before they attained a similar thickness as the large-reduction samples. Figures $2(a)$ and $(b)$ show the core images obtained from the small-reduction/pass and the large-reduction/pass rolling series respectively. It is clear that, at the same $\sum_{i} \boldsymbol{N}_{i} / h_{0}$ (total reduction/original thickness ratio) level, the large-reduction/

(a)

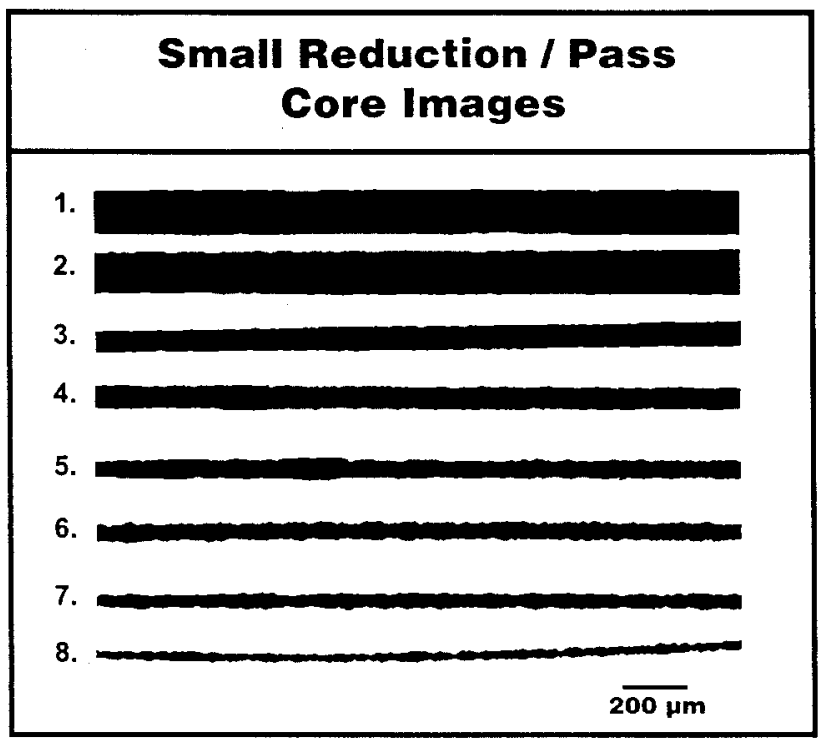

(b)

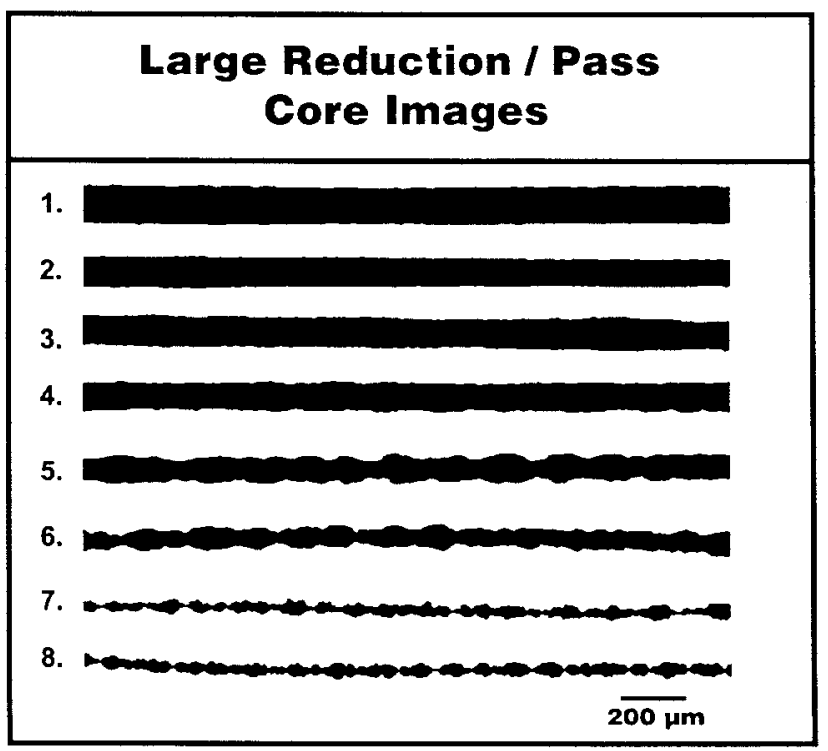

Figure 2. Core images obtained from $(a)$ the small-reduction/pass $(5 \%)$ and $(b)$ the largereduction/pass $(25 \%)$ rolling series. The large-reduction/pass rolling schedule produced much higher 'sausaging' magnitude than the small-reduction/pass rolling schedule. 


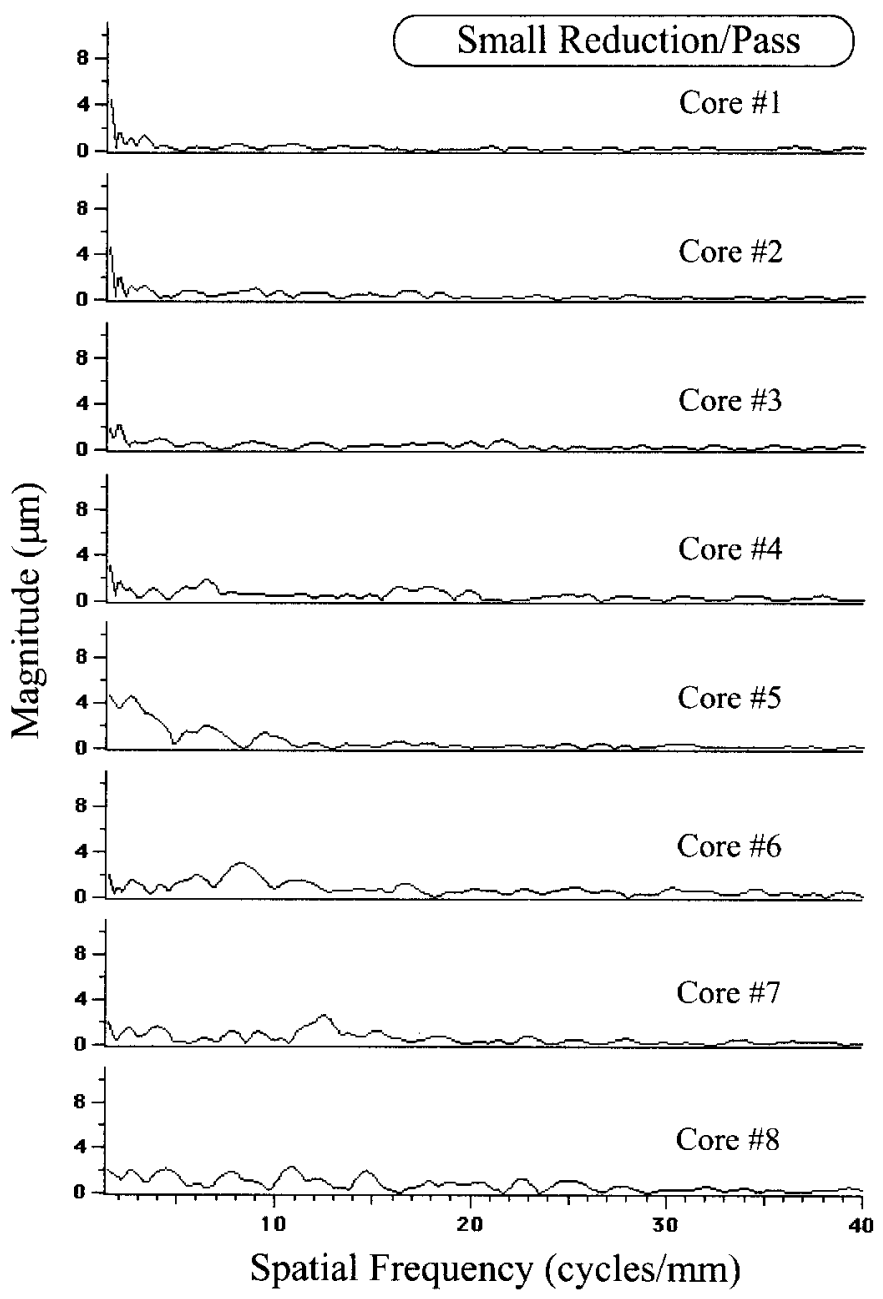

Figure 3. FFT analysis results for the small-reduction/pass (5\%) case. The FFT analyses were performed after measuring the core thickness variations along the tape length from the core images shown in figure $2(a)$.

pass rolling schedule produced a higher 'sausaging' magnitude than did the smallreduction/pass rolling schedule. This is consistent with earlier observations (Schoenfeld et al. 1996b).

\subsection{Fast Fourier transform frequency analyses}

After measuring the core thickness variations along the tape length from the core images shown in Figure 2(a) for the small-reduction/pass case, the FFT analyses were performed (Figure 3). Experimental data showed that the 'instability', or the significant growth of $\Delta / d_{\mathrm{c}}\left(\Delta \mathrm{v}\right.$ is the maximum magnitude of variation, and $d_{\mathrm{c}}$ is the current core thickness), was initiated after core 4 and continued to grow at later passes. Table 1 shows the wavelengths associated with the highest variation magnitude for the small-reduction/pass case.

After measuring the core thickness variations along the tape length from the core images shown in Figure 2(b) for the large-reduction/pass case, FFT analyses were 
Table 1. Wavelengths associated with the highest variation magnitude for the small-reduction/pass case.

\begin{tabular}{lccl}
\hline Core & $\begin{array}{c}\text { Wavelength } \lambda \\
(\mu \mathrm{m})\end{array}$ & $\begin{array}{c}\text { Average core thickness } d_{\mathrm{c}} \\
(\mu \mathrm{m})\end{array}$ & $\lambda / d_{\mathrm{c}}$ \\
\hline 4 & 154 & 73.2 & 2.1 (initiation) \\
$5^{a}$ & 151 & 58.2 & 2.6 \\
6 & 122 & 55.2 & 2.2 \\
7 & 80 & 45.1 & 1.8 \\
8 & 92 & 26.5 & 3.5 \\
\hline
\end{tabular}

${ }^{a}$ After taking out the first peak that produced by only one single long-wavelength variation.
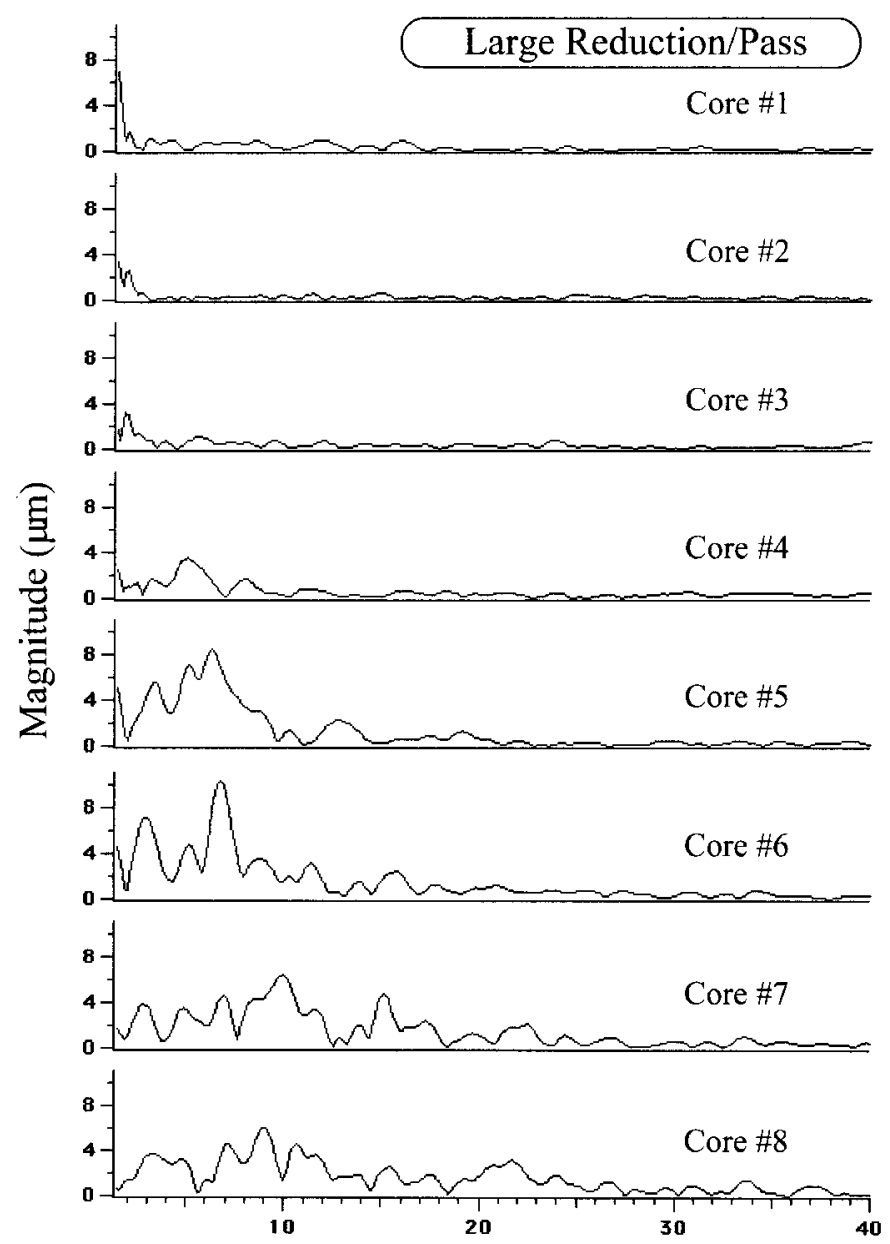

Spatial Frequency (cycles/mm)

Figure 4. FFT analysis results for the large-reduction/pass (25\%) case. The FFT analyses were performed after measuring the core thickness variations along the tape length from the core images shown in figure $2(b)$. 
Table 2. Wavelengths associated with the highest variation magnitude for the large-reduction/pass case.

\begin{tabular}{lccl}
\hline Core & $\begin{array}{c}\text { Wavelength } \lambda \\
(\mu \mathrm{m})\end{array}$ & $\begin{array}{c}\text { Average core thickness } d_{\mathrm{c}} \\
(\mu \mathrm{m})\end{array}$ & $\lambda / d_{\mathcal{c}}$ \\
\hline 4 & 192 & 89.7 & 2.1 (initiation) \\
5 & 158 & 78.7 & 2.0 \\
6 & 147 & 59.4 & 2.5 \\
7 & 101 & 30.2 & 3.3 \\
8 & 110 & 32.2 & 3.4 \\
\hline
\end{tabular}

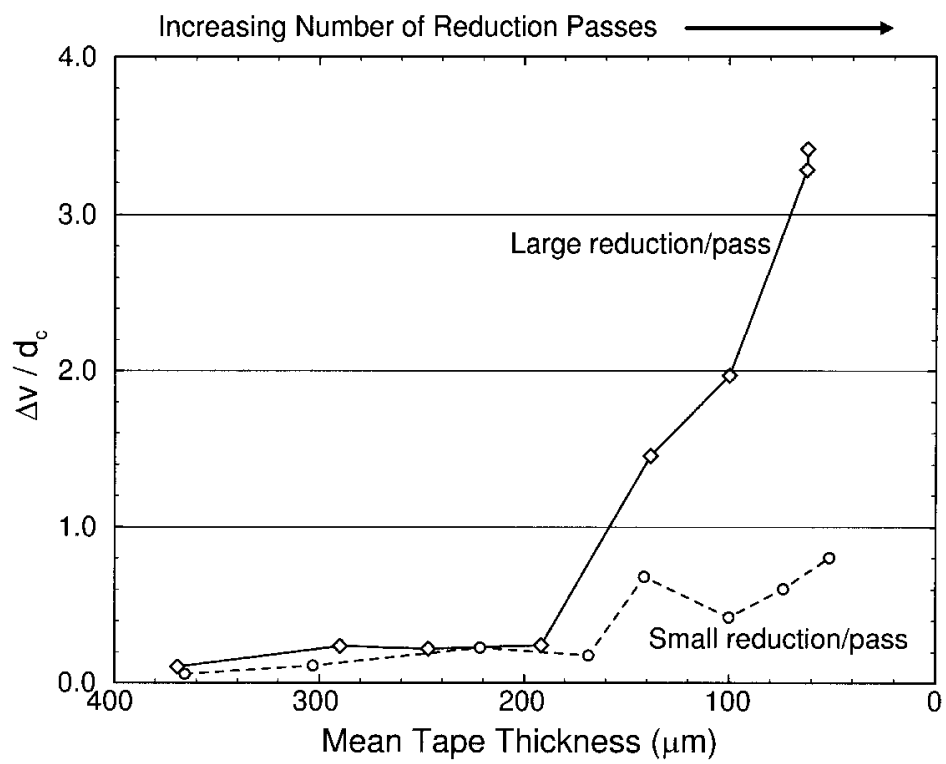

Figure 5. Plot of thickness variance/mean core thickness for the small-reduction/pass (5\%) and large-reduction/pass (25\%) cases. The large-reduction/pass case resulted in faster growth in variance magnitude.

performed (figure 4). Experimental data showed that the 'instability' or the significant growth of $\Delta / d_{c}$ began after core 4 and continued to grow at later passes, although at a much faster pace than the case of small reduction/pass. Table 2 shows the wavelengths associated with the highest variation magnitude for the large-reduction/pass case.

Tables 1 and 2 indicate that the instability initiation strain (i.e. the average core thickness at initiation) and the most significant wavelengths $\lambda / d_{\mathrm{c}}$ are found to be almost the same for both cases.

Figure 5 shows the plot of thickness variance/mean core thickness for the smallreduction pass case and for large-reduction/pass case. It is clear that a large reduction/pass resulted in faster growth in variance magnitude.

\subsection{Some earlier experimental results}

In one of our earlier studies (Schoenfeld et al. 1996b), some interesting and related phenomena were documented. Looking at figure 9 below, it is shown that as a result of texture development, the core material will eventually 'lock up' and can 


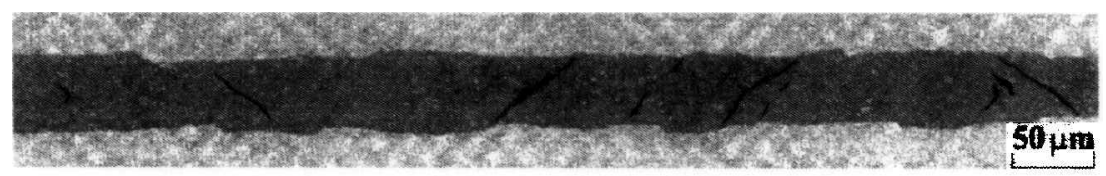

Figure 6. Optical micrograph of a longitudinal section from an OPIT-processed tape. In this tape, texture developed too fast, giving rise to high stresses followed by large cracked sections in the core. (Figure taken from Schoenfeld et al. (1996b).)

deform thereafter only at large stress levels. The rolling schedule with a large reduction tends to produce a higher hydrostatic pressure within the core and to result in faster texture growth. If the stresses rise too rapidly in the core, profuse macroscopic cracking, as shown in figure 6 , will become the dominant mode of deformation. If rolling were stopped here, these transverse cracks would ultimately result in a decrease in the superconducting transport properties. Even if, in an attempt to heal the cracks, further rolling is prescribed, the large blocky structures resulting from the cracked state could set the stage for other types of instability. Non-uniform densification or even discontinuous sections of the core could result.

Figure 7 shows micrographs of longitudinal sections from the four processing conditions for silver-clad tape after rolling to true compressive strains on the order of $250 \%$. A strong inverse correlation between reduction/pass and core dimensional uniformity was observed. The same effect was observed for the lower-strength aluminium-clad tapes, with core instabilities developing at somewhat smaller strains, and being more pronounced at final reductions (Schoenfeld et al. 1996b). As seen from the micrographs, the tape rolled by small reductions has a more uniform core-sheath interface and is more likely to maintain a smooth interface when subjected to further rolling. The tape rolled by large reductions has a much more pronounced 'sausage'-shaped core. Geometrical non-uniformities were linked to lower current densities (Parrell et al. 1993) and could be initiated from cracked states such as that shown in figure 6. From figures $7(b)$ and $(d)$, a rough estimate could be made that the most significant wavelength for
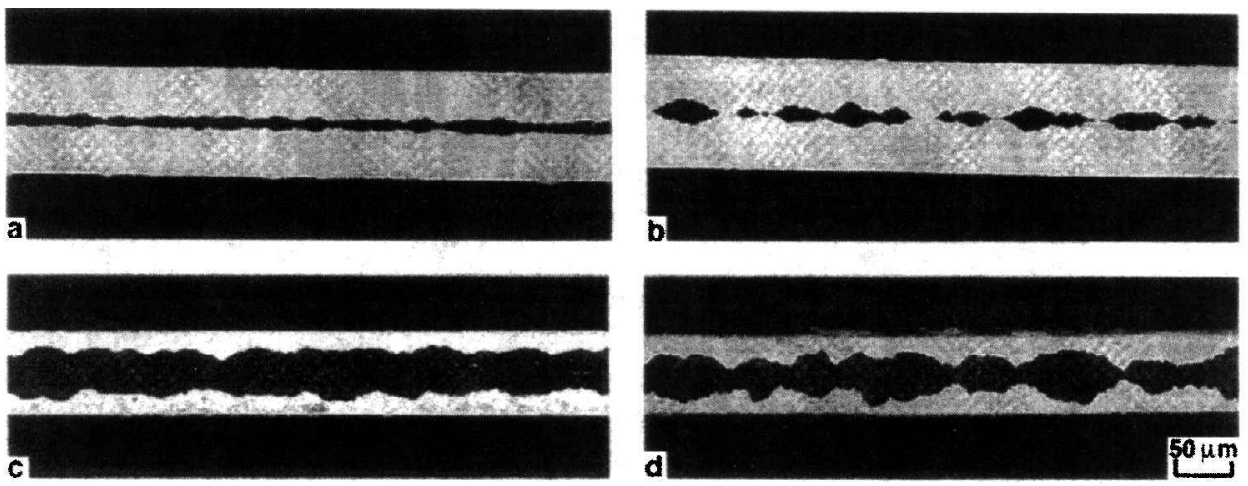

Figure 7. Light micrographs of longitudinal sections from silver-clad tapes showing inverse relationship between reduction per pass and core uniformity for both thin and thick cores: $(a) 5 \%$ per pass, core fill factor of $0.2 ;(b) 25$ per pass, core fill factor of $0.2 ;(c)$ $5 \%$ per pass, core fill factor of 0.6 ; (d) $25 \%$ per pass, core fill factor of 0.6 . (Figure taken from Schoenfeld et al. (1996b).) 

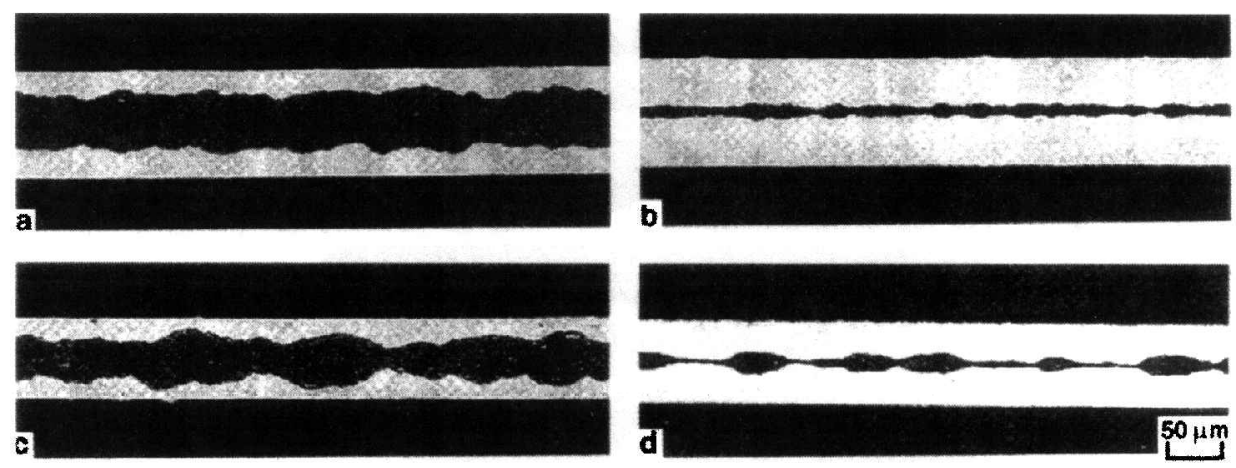

Figure 8. Light micrographs of longitudinal sections from silver- and aluminium-clad tapes rolled at 5\% per pass, showing the effect of relative core thickness and clad material on core uniformity: $(a)$ silver clad, core fill factor of 0.6 ; $(b)$ silver clad, core fill factor of $0.2 ;(c)$ aluminium clad, core fill factor of $0.6 ;(d)$ aluminium clad, core fill factor of 0.2 .

(Figure taken from Schoenfeld et al. (1996b).)

figure $7(d)$ is of the order of $(2-3) d_{c}$, and the wavelength for figure $7(b)$ is of the order of $(4-7) d_{\mathrm{c}}$. Another observation is that, comparing figure $7(b)$ with figure $7(d), \Delta \mathbf{v} / d_{\mathrm{c}}$ for the case in which the fill factor is $20 \%$ is larger than that for the case in which the fill factor is $60 \%$, since the former is apparently larger than unity and the latter is smaller than unity.

The positive effect of stronger clad material on core uniformity can be observed experimentally by comparing silver-clad (figures $8(a)$ and $8(b)$ ) with aluminium-clad (figures $8(c)$ and $8(d)$ ) tapes. Work-hardened yield strengths for $99.99 \%$ silver and 1100 aluminium are approximately $300 \mathrm{MPa}$ and $180 \mathrm{MPa}$ respectively. The stronger silver clad resulted in more uniform core geometries at large reductions. In fact, the low-fill-factor aluminium-clad tape is nearly discontinuous. The most significant wavelength for the aluminium-clad case with a fill factor of $60 \%$ (figure $8(c)$ ) is roughly estimated to be of the order $3 d_{\mathrm{c}}$, and for the aluminium-clad case with a fill factor of $20 \%$ (figure $8(d)$ ) to be of the order of $(5-8) d_{\mathrm{c}}$. Also, comparing figure $8(c)$ with figure $8(d), \Delta \mathbf{v} / d_{\mathrm{c}}$ for a fill factor of $20 \%$ is larger than that for a fill factor of $60 \%$.

\subsection{Summary of experimental observations}

The major experimental observations and FFT analysis results on the growth of interface instabilities (sausaging effects) can be summarized as follows.

(1) For a silver clad with a fill factor of $60 \%$, both the $25 \%$ reduction/pass and the $5 \%$ reduction/pass cases start to lose stability at almost the same compressive strain (i.e. the same thickness reduction level). The most significant (i.e. with the largest magnitude) wavelengths for both the $25 \%$ reduction/ pass and 5\% reduction/pass cases at the initiation are almost the same. The large-reduction/pass case (25\% per pass) has a much faster magnitude growth rate against reduction strain than does the small-reduction/pass case (5\% per pass). A similar trend is observed for other fill factors (i.e. $20 \%$ ) and/or other clad materials (i.e. aluminium); a smaller reduction/pass produces more uniform cores at larger reductions. 
(2) For the $5 \%$ reduction/pass with a fill factor of $20 \%$, the aluminium-clad case has a faster magnitude growth rate against reduction strain than does the silver-clad case. A similar trend is found with the 5\% reduction/pass rolling condition with a fill factor of $60 \%$; stronger clad material (i.e. silver, with a higher work hardening rate) results in more uniform cores at larger reductions.

(3) Smaller fill factors seem to result in faster growth in the normalized core variance $\Delta v / d_{\mathrm{c}}$ at the same compressive (rolling) strain of the tape.

(4) For the silver-clad case with a fill factor of $60 \%$ the most significant (i.e. with the largest magnitude) wavelengths for $25 \%$ reduction/pass and $5 \%$ reduction/pass cases at (and right after) the instability initiation are both around $2.1 d_{\mathrm{c}}$. For a fill factor of $20 \%$, the most significant (i.e. with the largest magnitude) wavelengths after the instability initiation appear to have larger $\lambda / d_{\mathrm{c}}$ ratios (estimated to be $\lambda \approx(4-7) d_{\mathrm{c}}$ in figure $7(b)$, and $\lambda \approx(5-8) d_{\mathrm{c}}$ in figure $8(d))$.

\section{$\S 3$. TAPe-Rolling MOdelling and simulations}

\subsection{Bifurcation analyses}

Treating the core and the clad as incompressible plastic materials, Steif (1986, 1987) established a procedure and the closed-form solutions to deal with the interface instabilities in bimaterial metal forming. As a first-order approximation, we treat both the clad material and the core as incompressible plastic materials and seek to gain some insight into the general trends in the interface instability development.

Figure $9(a)$ shows the uniaxial stress-strain curves used in our analysis, where a von Mises isotropic yield condition is assumed. The oxide core will experience some material locking at larger strains due to texture development after rolling (Asaro et al. 1992, Schoenfeld et al. 1996a) (see figure 9(b)). Since we shall be concerned with the initiation of interface instabilities using bifurcation analysis, the stress-strain curves for oxide at strains less than $70 \%$ will be described as those shown as solid curves in figure 9. Two clad materials, namely silver and aluminium, will be considered here. The material properties shown in figure 9 are described in Table 3.

Using these material properties, and concerning only the symmetric bifurcation mode (i.e. sausaging mode), bifurcation analyses were performed and the results are shown in figures 10 and 11. For details on how to obtain the bifurcation results, see Steif $(1986,1987)$. A periodic interface instability in terms of a sinusoidal functional form is a natural outcome of solving the plane-strain compression boundary value problem.

Figure 10 shows the bifurcation analysis results using silver clad with two different fill factors, $60 \%$ and $20 \%$; three oxide densities are examined in both cases. The results show that, the higher the core density, the lower is the bifurcation (instability) strain. Comparing figures, $10(a)$ and $(b)$, the $20 \%$ fill factor cases show lower critical bifurcation strains (see the lowest peaks) and much higher $\lambda / d_{\mathrm{c}}$ ratio than the $60 \%$ fill factor cases.

Figure 11 shows the bifurcation analysis results using oxide 2 core with two different fill factors, $60 \%$ and $20 \%$; silver clad and aluminium clad are examined in both cases. The results show that the silver clad (with higher strain hardening) will initiate the instability at higher strains than the aluminium clad, regardless of the fill 


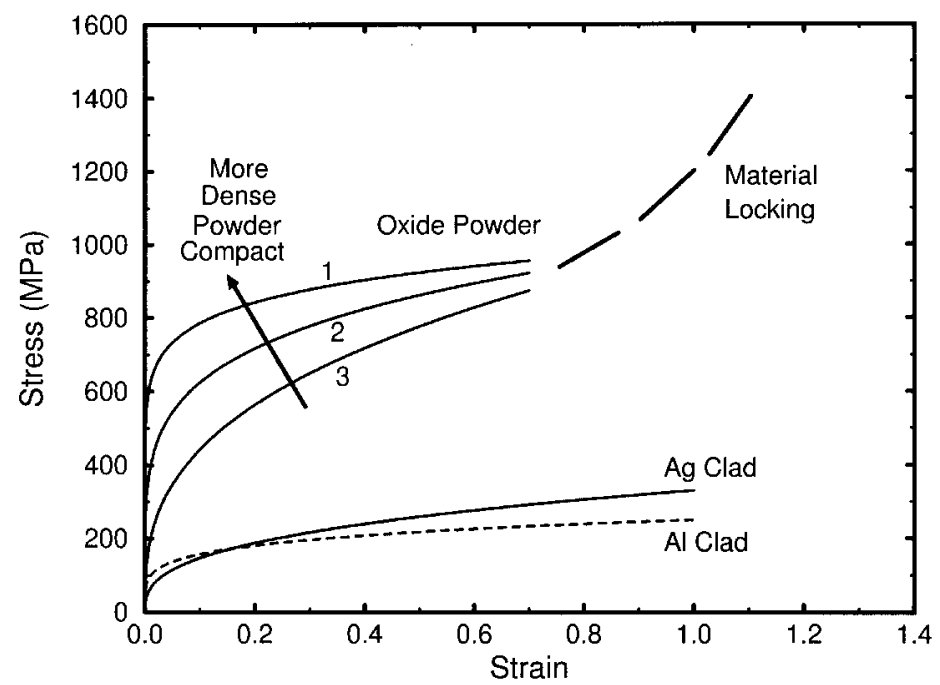

(a)

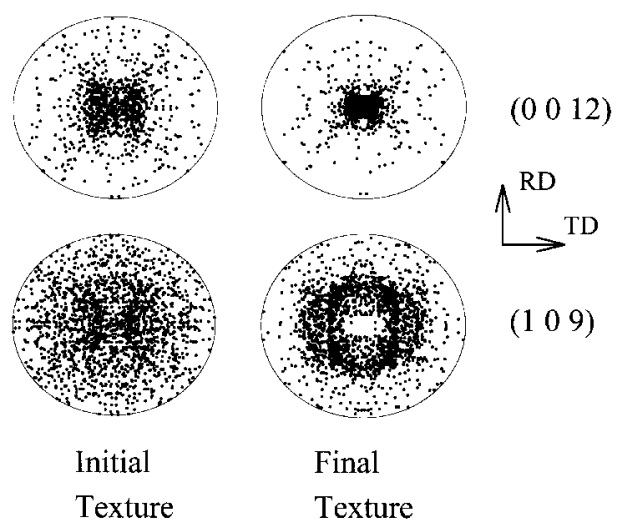

(b)

Figure 9. (a) Uniaxial stress-strain curves used in the analyses, and $(b)$ initial and final textures produced in modelling the channel die compression (rolling) of the BSCCO powder in an earlier study (Schoenfeld et al. 1996a). The texture development shown in $(b)$ is the main reason of the material locking at larger strains shown in $(a)$.

factors $(60 \%$ or $20 \%)$. It is also interesting to observe that the theory predicts similar critical wavelengths for the silver clad and the aluminum clad, if they have the same oxide fill factor. tions.

The bifurcation analysis results are consistent with the experimental observa-

(1) Bifurcation is strain controlled, so that the initiation strain is independent of the reduction/pass percentage.

(2) The lower fill-factor case starts the instability process earlier and is more likely to experience worse sausaging effects (i.e. higher $\Delta / d_{\mathrm{c}}$ ratio) than in the higher-fill-factor case. 
Table 3. Uniaxial stress-strain properties used in modelling $\left(\sigma=k \epsilon^{N}\right)$.

\begin{tabular}{lcl}
\hline & $k$ & \\
& $(\mathrm{MPa})$ & $N$ \\
\hline Oxide 1 & 990 & 0.1 \\
Oxide 2 & 990 & 0.2 \\
Oxide 3 & 990 & 0.35 \\
Ag clad & 330 & 0.35 \\
Al clad & 250 & 0.2 \\
\hline
\end{tabular}

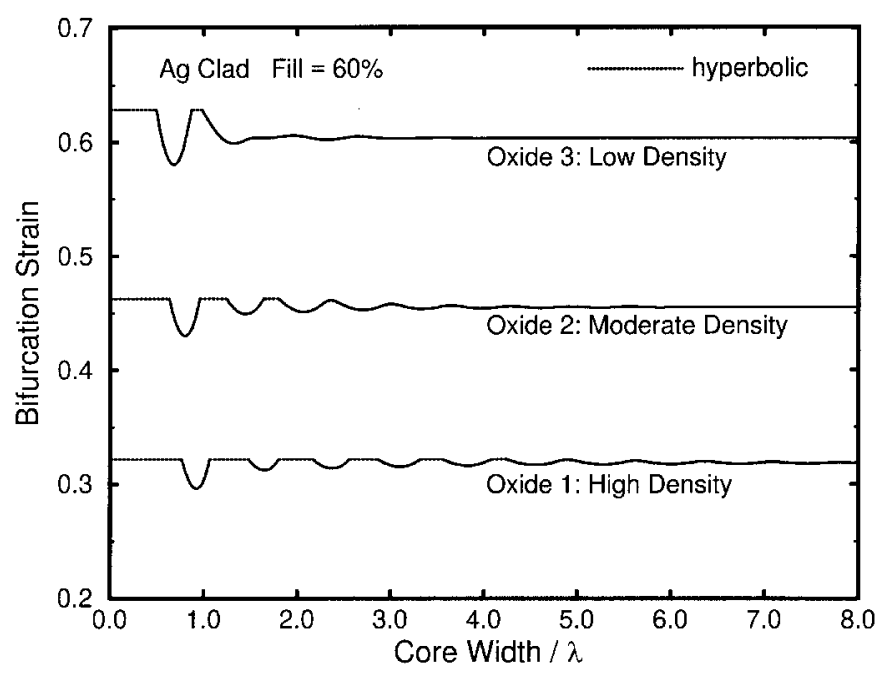

(a)

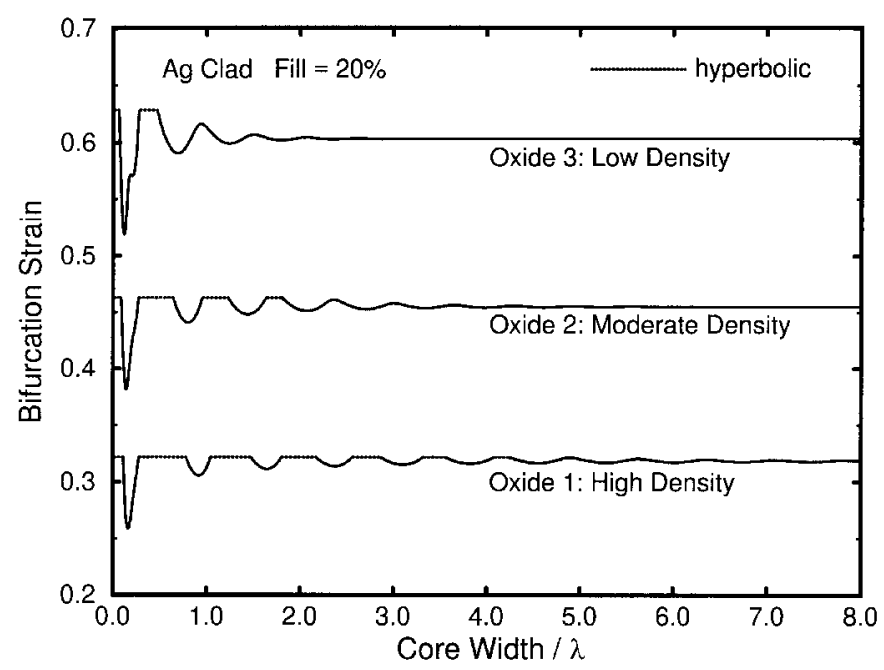

(b)

Figure 10. Bifurcation analysis results using silver clad with two different fill factors: $(a)$ $60 \%$; (b) $20 \%$. Three oxide densities are examined in both cases. 


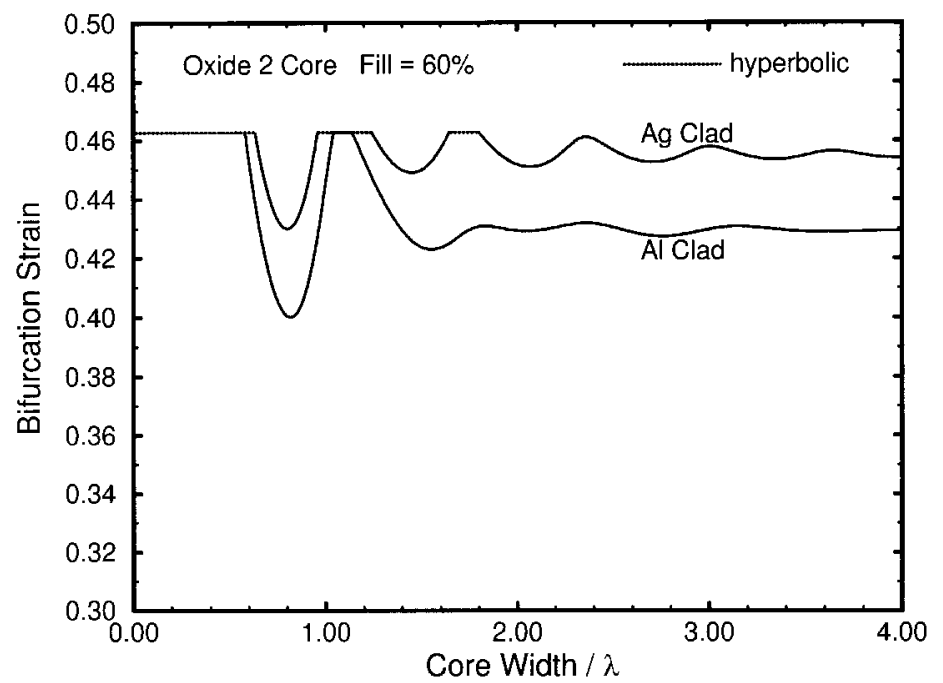

(a)

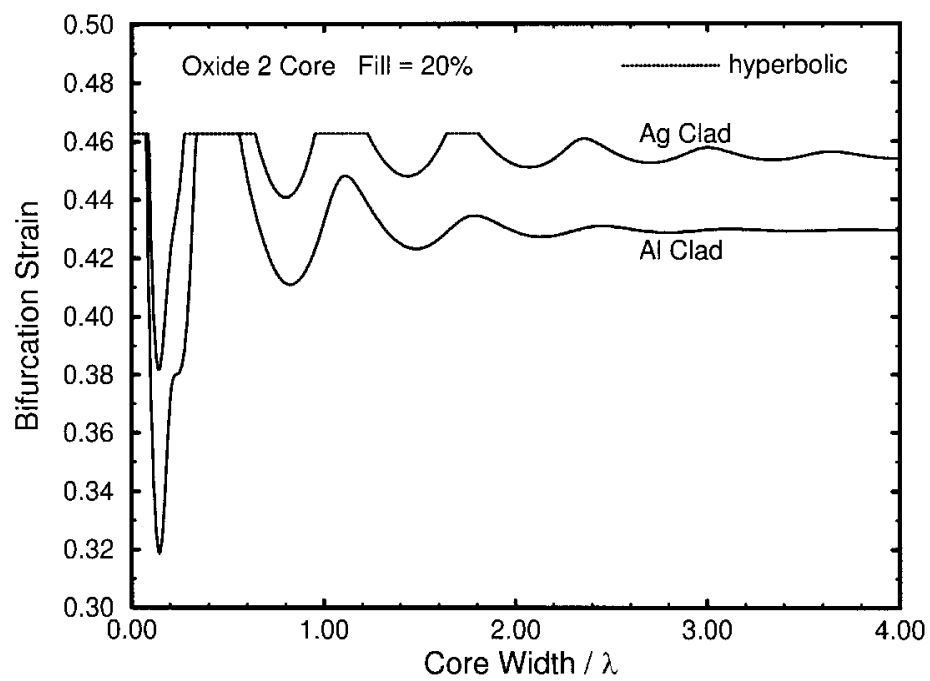

(b)

Figure 11. Bifurcation analysis results using oxide 2 core with two different fill factors: $(a)$ $60 \%$; (b) 20\%. Silver clad and aluminium clad are examined in both cases.

(3) The silver clad case starts the instability process later than the aluminiumclad case.

(4) The critical wavelengths for the $20 \%$ fill factor cases have much larger $\lambda / d_{\mathrm{c}}$ ratios $\left(\lambda \approx(6.1-8.3) d_{\mathrm{c}}\right)$ than the $60 \%$ fill factor cases $\left(\lambda \approx(1-1.5) d_{\mathrm{c}}\right)$.

\subsection{Finite-element modelling of tape rolling}

During the processing of superconducting tapes, the two-phase Ag-BSCCO workpiece, known as the OPIT conductor, obtains its final shape after being 
drawn down to wire from an initial composite billet. The wire is then flattened and elongated into tape by repeated passes between rollers with intermittent heat treatments and further rolling (Sandhage et al. 1991). Successful thermomechanical processing will produce long lengths of highly dense tapes with dimensionally uniform cross-sections and microstructures ideally aligned for optimal $J_{\mathrm{c}}$. Recent attempts at processing have met with mixed success (Haldar et al., 1992) and have relied heavily on trial-and-error experimentation with process parameters. New advances in material modelling (Schoenfeld et al. 1996a, b) have added insight into the macroscopic behavior of BSCCO aggregates subjected to various processing environments. The purpose of the current work is to understand the deformation environment and interface instability (sausaging) during what we feel are some of the more critical stages of processing; the final stages of rolling wire to tape. Such modelling has the capability of showing us how the process may be improved with a minimum of expensive trial-and-error experimentation.

During the rolling process, the workpiece is drawn through the roll gap by frictional interaction between the rolls and workpiece. As it moves through the roll gap, the workpiece is simultaneously compressed and sheared. Surface shear strains due to frictional interaction diminish towards the centre of the workpiece, while additional through-thickness shear strains may develop as a function of the workpiece geometry. Schoenfeld and Asaro (1996) calculated such through-thickness shear strain gradients in single phase workpieces for different roll-gap geometries. Asbeck and Mecking (1978) characterize the roll-gap geometry by a single nondimensional parameter $\zeta$ defined as the length of contact between the roll and workpiece normalized by the initial thickness of the workpiece prior to a particular pass. With reference to figure 1 , we define

$$
\zeta=\frac{2 l_{\mathrm{c}}}{h}=\frac{2 r \theta}{h}=\frac{2 r \cos ^{-1}(1-\Delta h / 2 r)}{h},
$$

which accounts for contact between the workpiece and both rolls. Schoenfeld and Asaro (1996) demonstrated that rolling conducted by maintaining a constant $\zeta$ rather than a constant $\Delta h$ or $\Delta h / h$, will maintain through-thickness deformation fields that scale with each subsequent pass. In other words, shear strains through the thickness of the workpiece will scale with $\zeta$ With this in mind, the entire roll-gap geometry can now be characterized by specifying two parameters: $\zeta$ and the relative BSCCO-to-tape-thickness ratio (fill factor). Note that the relative BSCCO ratio may increase or decrease with continued rolling (also depending on the rolling parameters), but the current work can still be considered generally, without taking this into account.

In order to resolve the rolling environment that the BSCCO core would encounter during the final stages of rolling the OPIT wire to tape, we have constructed a simple finite-element model using the ABAQUS finite-element package. The model contains anywhere from 1000 to 4400 plane-strain four-Gauss-point quadrilaterals depending on the particular roll gap calculation. A rigid roller draws the workpiece through the roll gap by frictional shear stresses which are not allowed to exceed the flow stress in the outer cladding material. This is controlled through the use of a sliding contact algorithm in the ABAQUS analysis package (ABAQUS Theory Manual Version 5.5 1995). Figure 1 shows the schematic rolling geometry of the conventional OPIT process. Since the workpiece has undergone substantial deformation by this time, the material interface between the silver and the core is 
considered to be perfectly bound; as such, no explicit interface properties are modelled; there is simply a discontinuity of material properties within the finite-element mesh.

The actual roll-gap geometries that typically occur during tape rolling are too long and narrow to appear coherently on one page; therefore, the actual finite-element meshes used to model the above roll gaps will not be shown here. A symmetry boundary condition is used along the centre of the workpiece.

The constitutive model chosen for the materials is a simple $J_{2}$ flow theory. This is a rather simplistic assumption when one considers the complex macroscopic behavior of BSCCO (Schoenfeld et al. 1996a) but justified for this particular process. Schoenfeld and Asaro (1996) accurately calculated the microstructures of rolled fcc materials post mortem from finite-element calculations using this exact assumption. Granted, fcc materials are less sensitive to textural hardening than the BSCCO oxide, but we believe the rolling process to be highly constrained. As such, any reasonable constitutive description should yield accurate resolution of the roll-gap deformation field.

Again, the uniaxial plastic stress-strain behavior shown in figure 9 is used for this simple model. The radius of the roller used in the calculations is $6.35 \mathrm{~cm}(2.5 \mathrm{inch})$ and the total thickness of the initial bimaterial tape will be $0.5 \mathrm{~mm}$. Two different relative BSCCO thickness ratios will be examined. In the first, the BSCCO represents 0.20 of the tape thickness, and in the second the oxide accounts for 0.60 of the tape thickness.

To study the interface instability (sausaging effects), here we are mainly concerned with the symmetric mode so that only the upper half of the geometry shown in figure 1 needs to be modelled. The various wavelength of defects will be excited by varying the flow strength of the oxide core down the length $(x)$ of the workpiece. In the bifurcation analyses, a periodic interface instability in terms of sinusoidal functional form is found to be a natural outcome of solving the plane-strain compression boundary value problem. The perturbation in flow strength is thus assumed to be periodic with a given wavelength $\lambda$ and a small variation amplitude $\Delta \sigma$ :

$$
\sigma_{\mathrm{y}}=\sigma_{0}+\Delta \sigma \sin \left(\frac{2 \pi x}{\lambda}\right) \text {. }
$$

Each defect would then be subjected to different amounts of thickness reduction by rolling. The resulting displacement fields will show greater sensitivity to certain defect wavelengths, so that a critical wavelength could be determined from the one that produces the largest interface variation. In this study, the perturbation magnitude $\Delta \sigma$ is taken as $0.02 \sigma_{0}$ in all cases.

Figure 12 shows the plot of the bimaterial interfaces after rolling for the case with oxide 1 as core, silver clad, 20\% fill factor and $25 \%$ reduction/pass. Discrete wavelengths were taken for $\lambda=(0.5-6.5) d_{\mathrm{c}}^{0}$, with a $0.5 d_{\mathrm{c}}^{0}$ increment. Here $d_{\mathrm{c}}^{0}$ is the original core thickness before rolling. If we examine the magnitudes of the resultant interface variations (cf. figure 12 ), the highest magnitude, $\Delta \boldsymbol{v} / d_{\mathrm{c}} \approx 5.95 \%$, is found with $\lambda=3.5 d_{\mathrm{c}}^{0}=6.1 d_{\mathrm{c}}$, where $d_{c}$ is the core thickness after the rolling. Similarly, for other cases, the critical wavelengths can be computationally determined, and the results will be summarized in the next section. It is also interesting to observe that, after the rolling, the strain field will be non-uniform within the bimaterial tape. Figure 13 shows the distribution profile of the equivalent plastic strain within a rolled tape section for the $\lambda=3.5 d_{\mathrm{c}}^{0}=6.1 d_{\mathrm{c}}$ case. 


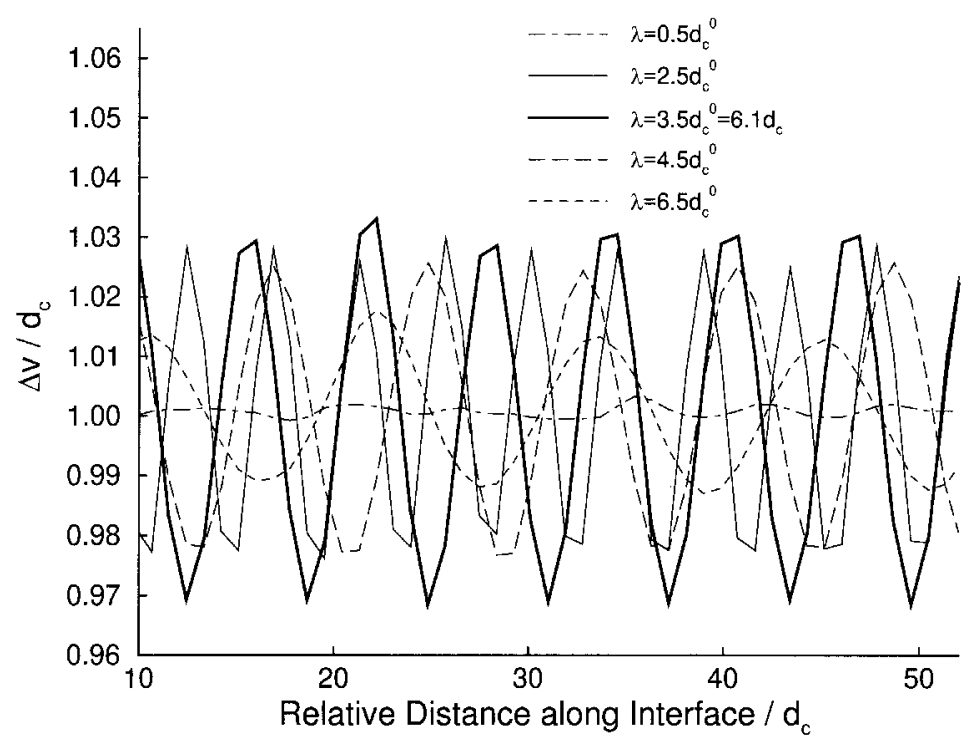

Figure 12. Plot of the bimaterial interfaces after rolling for the case with oxide 1 as core, silver clad, $20 \%$ fill factor and $25 \%$ reduction/pass. Discrete wavelengths were taken between $\lambda=(0.5-6.5) d_{\mathrm{c}}^{0}$, with a $0.5 d_{\mathrm{c}}^{0}$ increment (five wavelengths are shown here), where $d_{c}^{0}$ is the original core thickness before rolling.

\subsection{Summary of theoretical results and comparison with experimental results}

Table 4 is a summary of the results obtained. The finite-element model predicts quite accurately the critical wavelengths, while the bifurcation analyses (assuming a very simple plane-strain compression configuration) are reasonably close. All the qualitative trends obtained by the theoretical results are consistent with the experimental observations and FFT analysis summarized in $\S 2$.

Taking the magnitude of interface variations from table 4 and expressing them in terms of the original core thickness, $\boldsymbol{\Delta}^{*}$ (oxide $1 / \mathrm{Ag}$ ) at $5 \%$ reduction/pass is $0.31 \%$; and 5 times $0.31 \%$ is $1.55 \%$. On the other hand, $\boldsymbol{\Delta}^{*}$ (oxide $1 / \mathrm{Ag}$ ) at $25 \%$ reduction/pass is $1.40 \%$. Suppose that we could add the highest magnitudes from each rolling pass together, after five passes at $5 \%$ reduction/pass the total magnitude would be of the same order (if not larger) as that of the $25 \%$ reduction/pass case. As discussed earlier, smaller reduction/pass would not delay the initiation of the interface instability. However, the random disruption of the interface variation by the many rolling steps, with critical wavelengths very close to each other between adjacent rolling steps, will probably reduce the overall sausaging magnitude. It is similar to compare $5 \sin (x)$ with $\sum_{i=1}^{5} \sin \left(x+2 \pi a_{i}\right)$ where $a_{i}(i=1,2, \ldots, 5)$ are random numbers between 0 and 1 . Figure 14 shows the comparison with two different groups of $a_{i}$. Apparently, random summation with similar wavelengths and magnitudes will tend to reduce (average out) the interface variation.

We now list our findings in this study.

(1) The good correlation between theoretical results and experimental observations strongly suggests that the 'sausaging' effect is caused by the interface instability between the core and clad (sheath) materials.

(2) The critical ratio $\lambda^{*} / d_{c}$ is sensitive to the fill factor only. It is insensitive to the reduction/pass percentage, and rather insensitive to the core and 


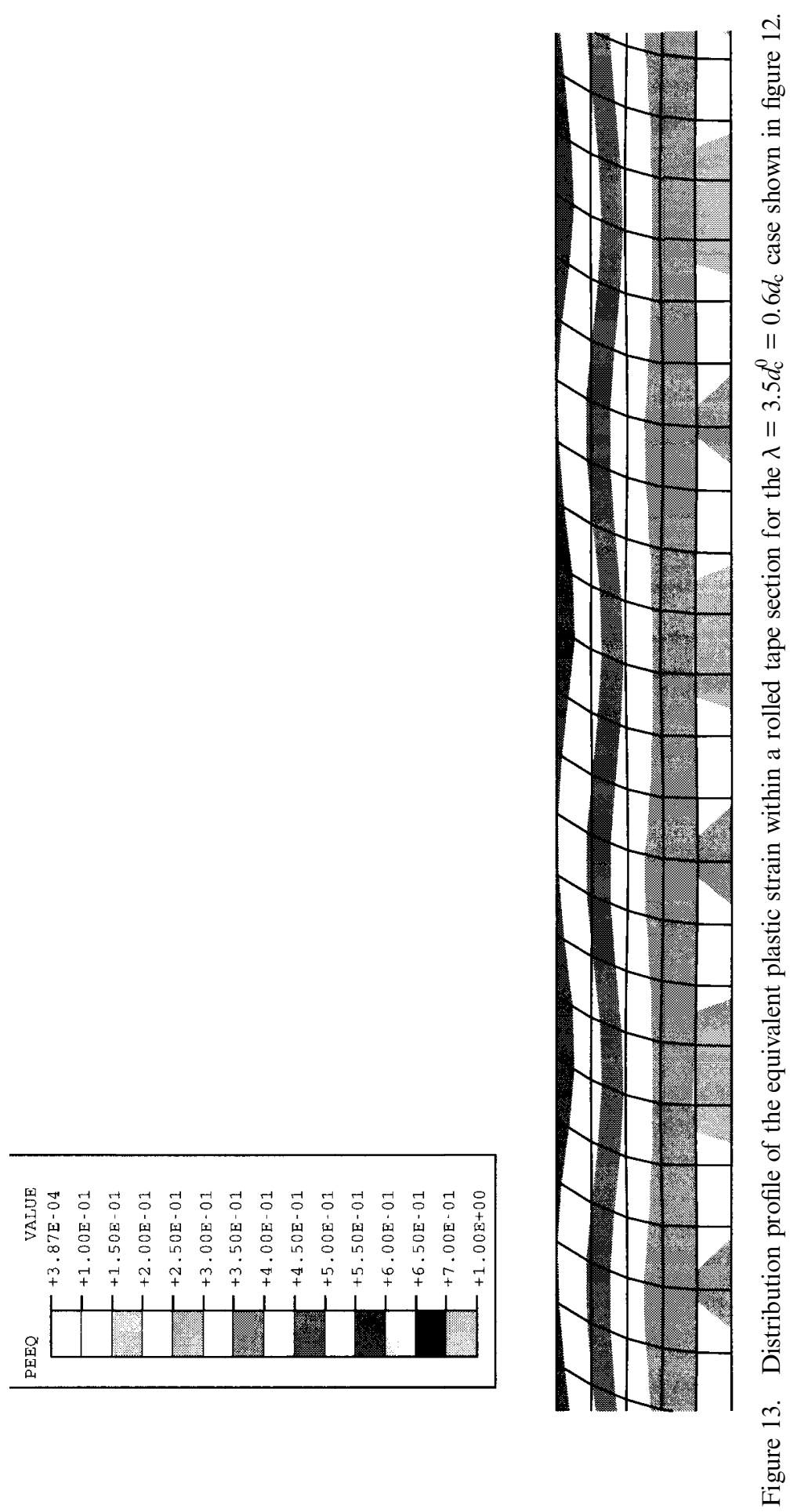


M. Dao et al.

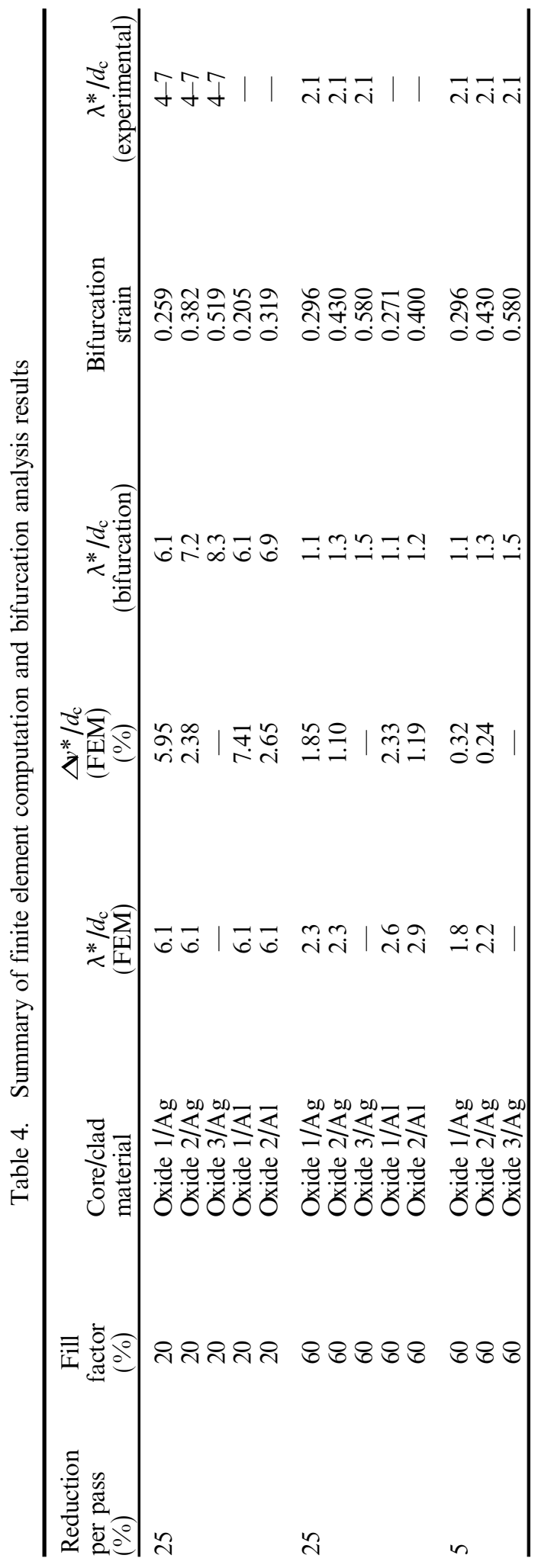




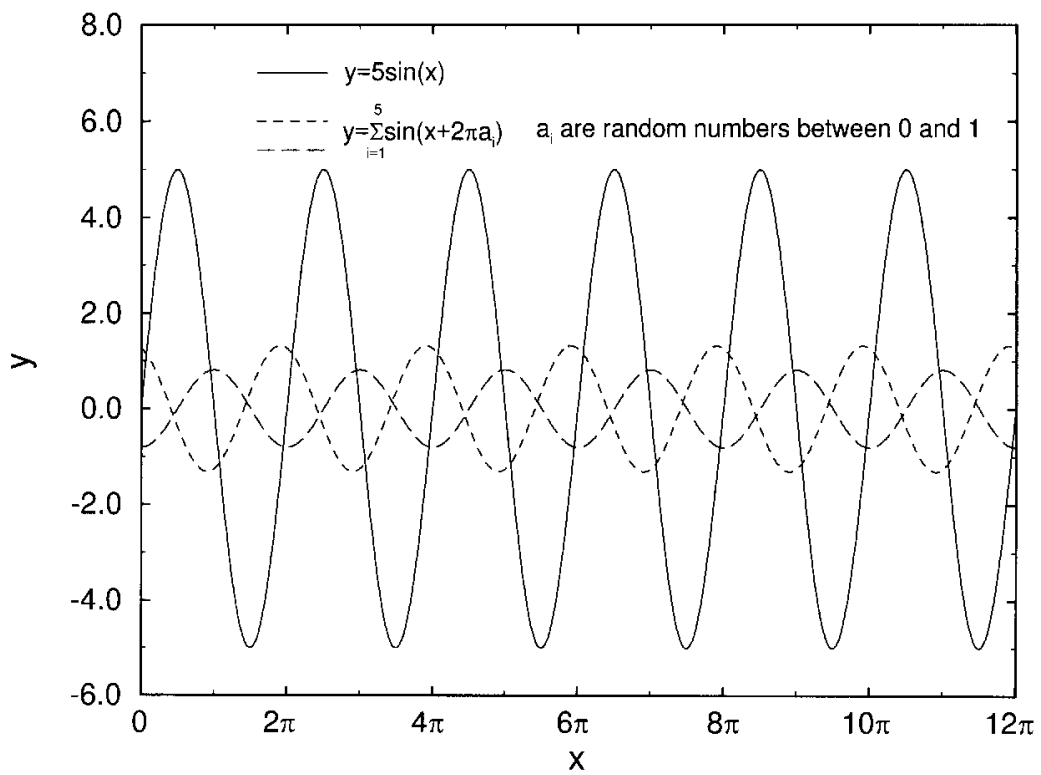

Figure 14. Comparison of the variation magnitudes of $5 \sin (x)$ and $\sum_{i=1}^{5} \sin \left(x+2 \pi a_{i}\right)$, where two different groups of random $a_{i}$ are taken.

clad material properties. In other words, the relative tape geometry is the single dominating factor that affects the normalized critical wavenumber $\lambda * / d_{\mathrm{c}}$.

(3) A smaller reduction/pass, a higher initial core porosity, higher-hardening clad material and a larger core fill factor can reduce the normalized instability magnitude $\Delta / d_{\mathrm{c}}$ at the same tape reduction strain level.

(4) The reason for the smaller $\Delta / d_{c}$ magnitude in the smaller-reduction/pass case is not because the smaller reduction/pass can eliminate or delay the interface instability initiation. One of the important reasons is very likely to be the random disruption of the interface variation by the many rolling steps with critical wavelengths very close to each other between adjacent rolling steps.

\section{$\S 4$. Discussion}

The results show that interface instability is a natural outcome of the rolling process. The oxide core will undergo either 'sausaging' or macroscopic cracking at larger reductions eventually.

Whereas the interface instability is certain to be initiated, there are a number of ways to delay or disrupt the magnitude growth. A smaller reduction/pass, a higher initial core porosity, a higher-hardening clad material and larger core fill factor are found to be useful in this regard. On the other hand, recent studies also showed that a combined compression-shear stress state, through adjusting the roll-gap geometry and controlling the shearing directions between adjacent passes, can delay the 'material locking' and produce higher $J_{\mathrm{c}}$ values in the tapes than a pure compression stress state (Blumenthal et al. 1996; Schoenfeld et al. 1996b). 
The smaller reduction/pass can reduce the 'sausaging' magnitude quite effectively. However, if the reduction/pass is too small, then it is economically not viable. To find some better ways to disrupt effectively the progress of the 'sausaging' effect is indeed a challenging task. The current study has provided us with useful and efficient quantitative tools in analyzing the tape-rolling processes along this direction. For example, to insert packing materials in certain rolling passes can change the 'effective' core fill factor between no-packing and with-packing passes and therefore change the wavelengths between the adjacent rolling passes. If designed carefully, this wavelength changes may effectively disrupt the magnitude growth of the interface instability. Further efforts are needed in this direction.

Carter et al. (1995) reported in their multifilament study that, unlike the predictions made in this study for single-filament tapes, stronger interface instabilities were found in higher fill factor tapes with certain multifilamentary architecture. There are more controlling parameters in the multifilamentary tape architectures, e.g., the dimensions of the original billets, the dimensions of the rebundling tube, and the number of rebundling steps. Careful studies to understand the relationship between the interface instability and these adjustable parameters are necessary.

Regarding the residual porosity in the oxide core, we note that the existence of porosity has two effects on the powder mechanical properties: Firstly it varies the stress-strain behaviour, and secondly it results in compressible plastic flow. In this study, assuming incompressible plastic deformation, we applied a simple model that accounts for the changes in stress-strain behaviour of the powder due to porosity; very good correlation between the theory and the experiments was obtained. On the other hand, oxide core compressibility may also have some important influences in the instability process during tape rolling. This is another interesting issue for future research.

\section{ACKNOWLEDGEMENTS}

M. D. and R. J. A. acknowledge financial support from the Naval Research Laboratory under contract N001493C2104, with Dr Patricia Paulette acting as technical monitor.

\section{REFERENCES}

ABAQUS Theory Manual Version 5.5, 1995 (Hibbitt, Karlsson and Sorenson).

Ahn, J. H., Ha, K. H. Lee, S. Y., Ko, J. W., Kim, H. D., and Chung, H, 1994, Jpn. J. appl. Phys., 33, L1298.

Ahzi, S., Asaro, R. J., and Parks, D. M., 1993, Mech. Mater., 15, 201.

Asaro, R. J., Ahzi, S., Blumenthal, W., and Digiovani, A., 1992, Phil. Mag. A, 66, 517. Asbeck, H. O., and Mecking, H., 1978, Materials Sci. Eng., 34, 111.

Blumenthal, W. R., Zhu, Y. T., Lowe, T. C., and Asaro, R. J., 1996, Physica C, 260, 33. Carter, W. L., Riley, Jr, G. N., Otto, A., Parker, D. R., Christopherson, C. J., Masur, L. J., and Buczer, D., 1995, IEEE Trans. appl. Supercond., 5, 1145.

Fischer, K., Schlafer, U., Rodig, C., Schubert, M., Hassler, W., Roas, B., Neu muller, H. W., and Jenovelis, A., 1995, Proceedings of EUCAS '95, Inst. Phys. Conf. Series

No. 148 (Bristol, UK: Institute of Physics), p. 363.

Haldar, P., Hoenn, J. G., Rice, J. A., and Motowidlo, L. R., 1992, Phys. Lett., 60, 495. Han, Z., Skov-Hansen, P., and Freltoft, T., 1997, Supercond. Sci. Technol., 10, 371.

Husek, I., Kovac, P., and Pachla, W., 1995, Supercond. Sci. Technol., 8, 617.

Karuna, M., Parrell, J. A., and Larbalestier, D. C., 1995, IEEE Trans. appl. Supercond., 5,1279 .

Korzekwa, D. A., Bingert, J. F., Podtburg, E. J., and Miles, P., 1994, Appl. Supercond., 2, 261. 
Lee, B. J., Parks, D. M., and Ahzi, S., 1993, J. Mech. Phys. Solids, 41, 1651.

Parks, D. M., and Ahz I, S., 1990, J. Mech. Phys. Solids, 38, 701.

Parrell, J. A., Dorris, S. E., and Larbalestier, D. C., 1993, Adv. Cryogenic Engng Mater., 40A, 193.

Sandhage, K. H., Riley, G. N., and Carter, W. L.,1991, J. Metals, 23, 21.

Schoenfeld, S. E., Ahzi, S., and Asaro, R. J., 1995, J. Mech. Phys. Solids, 43, 415.

Schoenfeld, S. E., Ahz I, S., Asaro, R. J., and Blumenthal, W. R., 1996a, Phil. Mag. A, 73, 1565.

Schoenfeld, S. E., Asaro, R. J., Ahzi, S., Bingert, J. F., and Willis, J. O., 1996b, Phil. Mag. A, 73, 1591.

Schoenfeld, S. E., and Asaro, R. J., 1996, Int. J. Mech. Sci., 38, 661.

Steif, P. S., 1986, Int. J. Solids Struct., 22, 1571; 1987, J. Appl. Metalworking, 4, 317.

Tanaka, Y., Matsumoto, F., Maeda, H., and Ishizuka, M., 1995, IEEE Trans. appl. Supercond., $\mathbf{5}, 1158$. 